\section{The statistical mechanical basis of the triple isotope fractionation relationship}

\section{J.A. Hayles ${ }^{1 *}$, X. Cao², H. Bao}

open 2 access

\section{Abstract}

doi: 10.7185/geochemlet.1701

Multiple stable isotope relationships have found a growing variety of uses in geochemistry and cosmochemistry. Approximations to the statistical-mechanical models for predicting isotope effects have led to the notion that mass fractionation laws are constrained to a "canonical" range of possible values. Despite previous work indicating that these mass fractionation exponents can be highly variable, the concept of a constant relationship remains common. In this study, we demonstrate generically that the mass fractionation exponent, $\theta$ can take any value for small fractionations and that these deviations are measurable. In addition, we chalue for small fractionatons and that the dn addition, we chacta a cally relevant haematite-water system.

Received 5 March 2016 | Accepted 20 July 2016 | Published 17 August 2016

\section{Introduction}

Despite roots in physical chemistry, the study of triple isotope relationships has found its most widespread and varied footing in the realm of geochemistry and cosmochemistry. Multiple stable isotope ratios have, among other uses, found utility as tools to elucidate photochemical (Farquhar et al., 2000; Bao et al., 2008) or magnetic isotope effects (Blum et al., 2014) and as an identifying marker for extraterrestrial materials (Clayton et al., 1973). Because of advances in analytical resolution, now more than ever, it is critical to understand the natural range of multiple isotope relationships to further these pursuits. The three isotope relationship was recently re-investigated for complex processes by Bao et al. (2015) using a broadly approximate treatment for elemental processes (i.e. equilibrium

1. Department of Earth Science, Rice University, 6100 Main St., Houston, TX 77005, USA

Corresponding author (email: Justin.a.hayles@rice.edu)

2. Department of Geology \& Geophysics, E235, Howe-Russell Geosciences Complex, Louisiana State University, Baton Rouge, LA 70803, USA and kinetic isotope effects). The intrinsic relationships among temperature $(T)$ isotope fractionation factor $(\alpha)$, and three-isotope exponent $(\theta)$ in mass-dependent elemental processes at equilibrium have not been explored theoretically.

The first accurate model for predicting the separation of isotopes for equilibrium processes was published independently by Urey (1947) and Bigeleisen and Goeppert-Mayer (1947; the B-GM-U model). Their development of the concept of reduced partition function ratio (RPFR or $\left.\left(s / s^{\prime}\right) f\right)$ allowed for the calculation of $\alpha$ between two species using only the harmonic vibrational frequencies for each degree of freedom and temperature.

$$
\alpha_{A-B}=\frac{\beta_{A}}{\beta_{B}}
$$

where $A$ and $B$ indicate different chemical species. Using oxygen as an example,

${ }^{18} \alpha_{A-B}=\left(\left[{ }^{18} O\right] /\left[{ }^{6} O\right]\right)_{A} /\left(\left[{ }^{8} O\right] /\left[{ }^{16} O\right]\right)_{B}$ and ${ }^{18} \beta_{A}=\left(\left[{ }^{18} O\right] /\left[{ }^{6} O\right]\right)_{A} /\left(\left[{ }^{8} O\right] /\left[{ }^{16} O\right]\right)_{\text {Atomic-O }}$ Under the harmonic approximation, $\beta$ can be calculated by

$$
\begin{gathered}
\beta_{\text {harm }}^{*}=\prod_{i}^{3 n-6(5)}\left(\frac{u_{i}^{*}}{u_{i}}\right)_{\text {TRPR }}\left(\frac{e^{-u_{i}^{*} / 2}}{e^{-u_{i} / 2}}\right)_{Z P E}\left(\frac{1-e^{-u_{i}}}{1-e^{-u_{i}^{*}}}\right)_{E X C} \\
u_{i}^{*}=\frac{h v_{i}^{*}}{k_{B} T}
\end{gathered}
$$

Here, $h$ is the Planck constant, $k_{B}$ is Boltzmann's constant and $T$ is temperature in kelvin, $v$ is the harmonic vibrational frequency for a specific vibrational degree of freedom and the product is taken over all vibrational degrees of freedom, the number of which is given by $3 n-6$ for non-linear molecules and $3 n-5$ for linear molecules where $n$ is the number of atoms in that molecule. The heavy isotope substituted state is indicated by "** and the lack of a superscript indicates the reference isotope (commonly the most abundant isotope). The harmonic contribution can be separated into the product of three terms: (a) the Teller-Redlich product rule (TRPR) term; (b) the zero-point energy (ZPE) contribution; and (c) non-zero point energy contributions (EXC) (Wolfsberg, 1972). Importantly, the $\beta$ in Equation 2 is modified with a subscript "harm" to indicate that this term only represents the contributions to isotope partitioning from the harmonic portions of molecular vibrations. The B-GM-U model is successful as it gives the dominant contribution to the value of $\beta$ to a point near or better than analytical precision for most isotope systems. However, for molecules bearing light isotopes, particularly hydrogen, contributions from anharmonicity, non-classical rotations and rotational-vibrational interactions become significant. Modifications of the B-GM-U model in the form of multiplicative terms have been developed to correct these inaccuracies. Here we use the term "contribution" to specifically indicate multiplicative terms. Each contribution ( $\mathrm{g}$, harmonic vibrational, anharmonic vibrational, rotational, imaginary etc.) is treated as having its own parameter, such as $\beta$, which is only a portion of the complete parameter. 


\section{Extension to Three Isotope Effects}

Transformation of a two-isotope model to a three-isotope model involves combining two independently calculated models that use the same reference isotope. A key aspect for the B-GM-U model is the mass dependence of isotope fractionation due to the mass dependence of vibrational frequencies. This relationship can be described by an exponent $\theta$ where

$$
\theta=\frac{\ln a_{A-B}^{\ddagger}}{\ln a_{A-B}^{*}}
$$

where "f" and " "" indicate the respective parameters for two different isotope ratios of the same element. Due to the use of the "low-u" (see Eq. 3) approximation of Equation 2, it was commonly believed that the value of the three isotope exponent was somewhat constant and later thought to be constrained to a finite, "canonical", range for mass-dependent processes which remains as an accepted idea (Swain et al., 1958; Matsuhisa et al., 1978; Young et al., 2002, 2016; Kaiser et al., 2004). Here, "canonical" refers to a $\theta$ value being within certain range represented by most of the observed cases and theoretical justifications (e.g., 0.50-0.5305 for the ${ }^{16} \mathrm{O}_{-17} \mathrm{O}^{18} \mathrm{O}$ system; Eiler et al., 2013).

Using a theoretical framework which represented strictly mass dependent scenarios, Skaron and Wolfsberg (1980) showed theoretically for oxygen isotope partitioning that $\theta$ can vary from positive to negative infinity for crossover scenarios, situations where the fractionation factor $\alpha$ for transitions from greater than 1 (heavier isotopes partitioned to A) to less than 1 (heavier isotopes partitioned to B) or vice-versa with changing temperature. Studies investigating these scenarios for sulphur (Deines, 2003; Otake et al., 2008) and hydrogen (Kotaka et al., 1992) have come to the same conclusion.

Analogously to the concept of $\beta$, Cao and Liu (2011) first introduced the concept of $\kappa$ defined as the one species analogue of $\theta$, or

$$
\kappa=\frac{\ln \beta^{\ddagger}}{\ln \beta^{*}}
$$

where $\beta$ is the one species fractionation factor for the two-isotope system. The complete value of $\kappa$ including all contributions can be calculated by

$$
\kappa_{\text {Complete }}=\frac{\Sigma_{i} \kappa_{i} \ln \beta_{i}^{*}}{\Sigma_{i} \ln \beta_{i}^{*}}
$$

where the sums are taken over all contributions whether they are from corrections (rotational, anharmonic etc.) to the B-GM-U model, from the imaginary frequency contribution for a transition state or from independent vibrational degrees of freedom. As long as all values of $\ln \beta$ have the same sign, such as for harmonic vibrational degrees of freedom, the value of $\kappa$ for a complex molecule is equal to the $\ln \beta$ weighted mean of the $\kappa$ values from each contribution. The relationship between $\kappa$ and $\theta$ is given by (Cao and Liu, 2011)

$$
\theta=\kappa_{A}+\left(\kappa_{A}-\kappa_{B}\right) \frac{\ln \beta_{B}^{*}}{\ln \alpha_{A-B}^{*}}
$$

From Equation 7, a large difference in the values of $\kappa$ between phases $\mathrm{A}$ and $\mathrm{B}$ allows for a larger variability in the value of $\theta$ particularly where $\alpha$ is near unity. The only scenario which would not allow the above conditions to occur for any system would be if the values of $\kappa$ and $\beta$ were related by an explicit function of one-another which could be universally applied to all species. This hypothetical case is invalid (see Fig. 1a) because the relationship between vibrational frequencies of differently substituted molecules is a function of not only the mass of the substituted atom, but also the molecular configuration, bond force constants, and masses of secondary, non-substituted atoms. Additional evidence against a universal $\kappa-\beta^{*}$ relationship can be found in studies implicating crossovers for $\theta$ values approaching infinity (Kotaka et al., 1992; Deines, 2003; Otake et al., 2008).

\section{Theoretical Values of $\kappa$}

The complete equation for the harmonic contribution to $\kappa$ for a single degree of freedom is given by

$$
\begin{aligned}
& \left(\ln \left(u^{\ddagger}\right)-\ln (u)\right)_{T R P R}+\left(u / 2-u^{\ddagger} / 2\right)_{Z P E}+ \\
& \kappa_{\text {harm }}=\frac{\left(\ln \left(1-e^{-u}\right)-\ln \left(1-e^{-u^{\ddagger}}\right)\right)_{E X C}}{\left(\ln \left(u^{*}\right)-\ln (u)\right)_{T R P R}+\left(u / 2-u^{*} / 2\right)_{Z P E}+} \\
& \left(\ln \left(1-e^{-u}\right)-\ln \left(1-e^{-u^{*}}\right)\right)_{E X C}
\end{aligned}
$$

which can be separated into terms that correspond to the contributions from TRPR, ZPE and EXC with each having their own mass dependence. At high temperatures, the contribution from energised states (EXC) dominates the harmonic $\kappa$. In this instance the values of $u$ become very small and

$$
\kappa_{H T L}=\frac{1 / m-1 / m^{\ddagger}}{1 / m-1 / m^{*}}
$$


where $m, m^{\ddagger}$ and $m^{*}$ are the masses of the light, middle and heavy isotopes respectively. Equation 9 serves as an upper bound of its value for diatomic molecules at all temperatures (e.g., $\kappa_{H T L} \approx 0.5305$ for the ${ }^{16} \mathrm{O}_{-}{ }^{17} \mathrm{O}-{ }^{18} \mathrm{O}$ system). At low temperatures, the contribution from ZPE dominates the harmonic $\kappa$. For a diatomic molecule under these conditions

$$
\kappa_{L T-D}=\frac{1 / \sqrt{\mu}-1 / \sqrt{\mu^{\ddagger}}}{1 / \sqrt{\mu}-1 / \sqrt{\mu^{*}}}
$$

where $\mu$ is the reduced mass $(\mu=m M /[m+M]$ for a diatomic molecule where $M$ is the secondary, non-substituted mass). In this case, the value of $\kappa$ decreases with increasing mass of the non-substituted atom (Cao and Liu, 2011). The limit of Equation 10 as the non-substituted mass approaches infinity is then

$$
\kappa_{L L}=\frac{1 / \sqrt{m}-1 / \sqrt{m^{\ddagger}}}{1 / \sqrt{m}-1 / \sqrt{m^{*}}}
$$

which serves as an unattainable lower boundary of $\kappa$ for diatomic molecules (e.g. $\kappa_{L L} \approx 0.5232$ for the ${ }^{16} \mathrm{O}_{-}{ }^{17} \mathrm{O}_{-}{ }^{18} \mathrm{O}$ system). Equation 10 is the same as the approximation to the mass fractionation law used by Swain et al. (1958) for triple-hydrogen system. Using the arguments of Swain et al. (1958), it is expected that polyatomic molecules will behave within or near the diatomic boundaries of $\kappa_{\text {harm }}$ presented here. For all calculated results for hypothetical diatomic molecules, the values of $\kappa_{\text {harm }}$ are found to not exceed the boundaries given by Equations 9 and 11.

Figure 1 displays the relationships between (a) $\beta$ and $\kappa$ and (b) $\theta$ and $\alpha$ for equilibrium between hypothetical diatomic molecules containing oxygen under the harmonic approximation with temperature plotted as colour. A key finding here is that the diatomic model allows $\theta$ to hold any value for small fractionations. Polyatomic molecules incorporate additional degrees of freedom which will weaken the relationship between $\kappa$ and $\beta$. Therefore, this result is a conservative estimate of the distribution of $\theta$. Much like values of $\alpha$ for a two isotope system the variability of $\theta$ becomes greater at lower temperatures. Because anharmonic corrections are small relative to the harmonic contribution and have a similar mass dependency, anharmonic corrections do not have a significant effect on the distribution of $\theta$ values. The effects of anharmonic corrections are addressed in more depth in the Supplementary Information. For kinetic isotope effects, the value of $\kappa$ for the transition states imaginary frequency contribution is also discussed in the Supplementary Information. The results indicate that values of $\theta$ for kinetic isotope effects are more variable and on average, but not strictly, lower than those of equilibrium isotope effects.
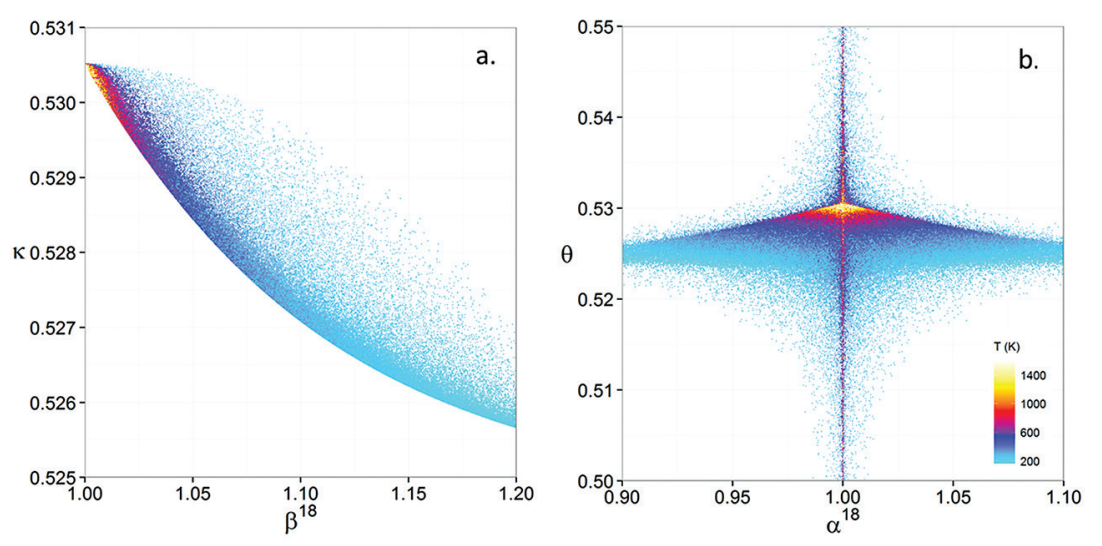

Figure 1 (a) $\beta^{18}$ vs. $\kappa$ and (b) Equilibrium $\alpha^{18}$ vs. $\theta$ plots for randomly selected hypothetical diatomic molecules under the harmonic approximation in the ${ }^{16} \mathrm{O}^{-17} \mathrm{O}-{ }^{-18} \mathrm{O}$ system. Temperature is plotted as colour on the same scale for both plots. The model uses $1,000,000$ evenly distributed randomly generated values for the secondary mass (0-300 AMU), the bond force constant $\left(0-\mathrm{K}_{U 1}\right)$, and temperature $\left(200-1600^{\circ} \mathrm{K}\right)$. The bond force constant for carbon monoxide $(\mathrm{CO})$ is used as an arbitrary upper limit (UL) for the bond force constant for diatomic oxygen species.

\section{Change/Difference in Cap-Delta}

Although $\kappa$ is expected to be bounded to a first-order approximation by the high temperature and lower limits as described above, the more common massdependent fractionation exponent, $\theta$, is not. What is needed is an additional descriptor for multiple isotope fractionation relationships that is bounded for small isotope effects.

Measured multiple isotope relationships are commonly expressed in the "cap-delta" notation.

$$
\Delta^{\ddagger} M=\ln \left(R_{\text {sample }}^{\ddagger} / R_{\text {standard }}^{\ddagger}\right)-C \ln \left(R_{\text {sample }}^{*} / R_{\text {standard }}^{*}\right)
$$

typically either in "per mille" or "per meg", where $C$ is a somewhat arbitrary constant and $R$ is the molar ratio of one isotope and a reference isotope of the same element. For example, $\Delta^{\ddagger} M$ becomes $\Delta^{17} \mathrm{O}$ for the ${ }^{16} \mathrm{O}^{17} \mathrm{O}-{ }^{18} \mathrm{O}$ system and $\ln \left(R_{\text {sample }}^{\ddagger} / R_{\text {standard }}^{\ddagger}\right)=\delta^{\prime 17} O$.

The change, or difference in cap-delta, i.e. $\Delta\left(\Delta^{\ddagger} M\right)$ through a fractionation process is then

$$
\Delta\left(\Delta^{\ddagger} M\right)=\ln \alpha_{A-B}^{\ddagger}-C \ln \alpha_{A-B}^{*}
$$

or reduced to more fundamental parameters 


$$
\Delta\left(\Delta^{\ddagger} M\right)=\left(C-\kappa_{B}\right) \ln \beta_{B}^{*}-\left(C-\kappa_{A}\right) \ln \beta_{A}^{*}
$$

Current analytical precision for $\Delta^{\ddagger} M$ can be as low as $0.002 \%$ depending on the element, technique, and species analysed allowing for the resolution of $\Delta\left(\Delta^{\ddagger} M\right)$ well within the natural range for many commonly explored light elements (e.g., for oxygen in Fig. 2). Using the ${ }^{16} \mathrm{O}^{17} \mathrm{O}-{ }^{18} \mathrm{O}$ system as an example, if the arbitrary upper limit of $\beta$ is given as 1.100 and using the boundaries of $\kappa$ proposed above for equilibrium (0.5232 to 0.5305), a potential range of $\Delta\left(\Delta^{17} O\right)$ is then about $\pm 0.7 \%$ o $(C=0.5305)$. The ends of this range are not realistic except at very low temperatures $\left(<<0{ }^{\circ} \mathrm{C}\right)$ where values of $\beta$ become larger and more variable and the lower boundary of $\kappa$ becomes more obtainable. A more realistic range for equilibrium between natural samples is likely in the vicinity of $\pm 0.3 \%$ o $(C=0.5305)$. This value is comparable to measured values for silica-water fractionation near 0 ${ }^{\circ} \mathrm{C}$ (Sharp et al., 2016). Kinetic isotope effects should have more variable $\Delta\left(\Delta^{17} O\right)$ values because of their more variable $\kappa$ values, however the range is expected to be similar to that of equilibrium isotope effects.

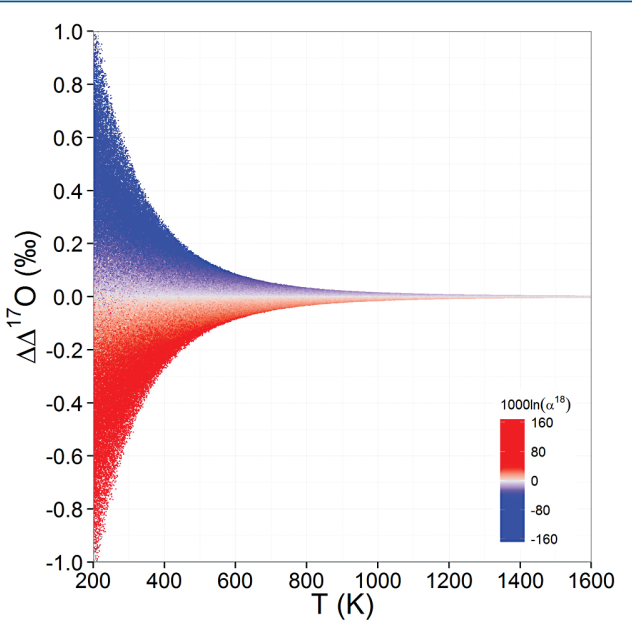

Figure 2 Temperature vs. $\Delta\left(\Delta^{17} O\right)$ plot for equilibrium between randomly selected diatomic molecules under the harmonic approximation in the ${ }^{16} \mathrm{O}_{-17} \mathrm{O}-{ }^{18} \mathrm{O}$ system. A $\mathrm{C}$ value of 0.5305 (HTL) is used for the $\Delta\left(\Delta^{17} O\right)$ definition. $1000 \ln \alpha^{18}$ is plotted as colour. The range of $\Delta\left(\Delta^{17} O\right)$ values in this plot is expected to be an overestimate for natural samples. The points in this model each correspond to a point in Figure $1 a$.

Regardless of the value of $C, \Delta\left(\Delta^{\ddagger} M\right)$ will approach a high temperature limit of 0 because both $\beta$ values approach a high temperature limit of 1.000 . We recommend the use of the high temperature limit of $\kappa$ for $C$ which will ensure that the $(C-\kappa)$ terms in Equation 14 remain positive. For sufficiently different $\beta$ values (i.e. non-small values of $\ln \alpha_{A-B}^{*}$ ) the sign of $\Delta^{\ddagger}\left(\Delta^{\ddagger} M\right)$ will be determined by the larger $\beta$. For positive $\ln \alpha_{A-B}^{*}$ values, $\Delta\left(\Delta^{\ddagger} M\right)$ will tend to be negative and vice-versa. This trend is not necessarily expected when $\alpha$ is near unity, particularly at low temperatures.

\section{Haematite-Water Fractionation}

For crossover scenarios, there is a guarantee that the determined $\alpha$ value will be near unity. In Bao and Koch (1999) haematite $\left(\mathrm{Fe}_{2} \mathrm{O}_{3}\right)$-water fractionation was measured over a range of near-surface temperatures. Nine samples are selected from this study for new triple oxygen isotope analysis. Details of the analysis and the tabulated results can be found in the Supplementary Information. The new calculated fractionation parameters with their uncertainties and best fits are illustrated in Figure 3.
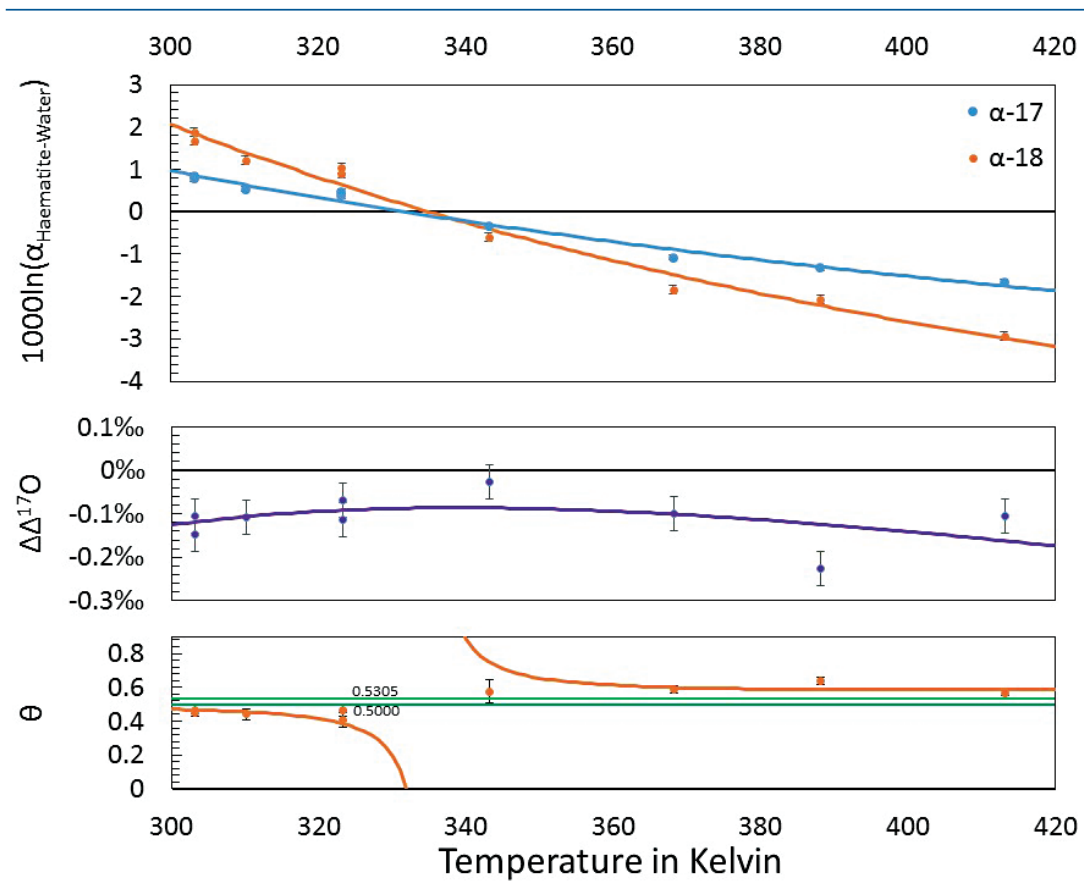

Figure 3 Three isotope fractionation parameters for haematite synthesis conducted by Bao and Koch (1999). Values are calculated from newly measured $\delta^{18} O_{\text {haematite }}$ and $\delta^{18} O_{\text {water }}$ values, and an assumed $\Delta^{17} \mathrm{O}(\mathrm{C}=0.528)$ of $0.02 \%$ for the water based on reported Baltimore tap water from Li et al. (2015). $\Delta\left(\Delta^{11} O\right)$ are reported using $C=0.5305$. The method for calculating uncertainties can be found in the Supplementary Information. Best fit curves for the In $(\alpha)$ values are second order polynomials of $1 / T$. The best fit curves for $\Delta\left(\Delta^{17} O\right)$ and $\theta$ are calculated from the fits to $\ln (\alpha)$. 
From the calculated haematite fractionation parameters, it is clear that the crossover temperature is different for $\alpha^{17}$ and $\alpha^{18}$. With one exception, every experimentally measured $\theta$ value exists outside of the typical "canonical" range of $\theta$ (approximately 0.5000 to 0.5305 ) to beyond $1 \sigma$ uncertainties. Despite the variability in $\theta, \Delta\left(\Delta^{17} O\right)$ values remain within a range of $-0.026 \%$ to $-0.226 \%$ within the theoretically predicted mass dependent range.

\section{Conclusions}

Using the simplest application of the B-GM-U model, it is shown theoretically that values of the mass fractionation exponent, $\theta$, are unbounded and particularly variable for small fractionations and at low temperatures. This is in contrast to the mass-fractionation exponent, $\kappa$, which is bounded by upper and lower limits. As a supplemental mass-fractionation descriptor, $\Delta\left(\Delta^{\ddagger} M\right)$ is suggested on a theoretical basis because it remains within a finite range for mass-dependent fractionations. The haematite-water system at near surface temperatures is cited as an example of deviations from the "canonical" range of $\theta$ for oxygen. These deviations are found to be consistent with a finite range of $\Delta\left(\Delta^{17} O\right)$. It is our opinion based on the results of this study that even for elemental processes the concept of a "canonical" range of $\theta$ values is not well founded and should be applied with caution.

\section{Acknowledgements}

Financial support is provided by US NSF grant EAR-1251824, China NSFC grant 41490635 to HB.

Editor: Eric H. Oelkers

\section{Additional Information}

Supplementary Information accompanies this letter at www.geochemicalperspectivesletters.org/article1701

Reprints and permission information is available online at http://www. geochemicalperspectivesletters.org/copyright-and-permissions

Cite this letter as: Hayles, J.A., Cao, X., Bao, H. (2017) The statistical mechanical basis of the triple isotope fractionation relationship. Geochem. Persp. Let. 3, 1-11.

\section{References}

BAO, H., CAO, X., HAYLES, J.A. (2015) The confines of triple oxygen isotope exponents in elemental and complex mass-dependent processes. Geochimica et Cosmochimica Acta 170, 39-50.
BAO, H.M., КосH, PL (1999) Oxyoen isotope fractionation in ferric oxide-water systems: Low temperature synthesis. Geochimica et Cosmochimica Acta 63, 599-613.

BaO, H.M., Lyons, J.R., ZHOU, C.M. (2008) Triple oxygen isotope evidence for elevated $\mathrm{CO}_{2}$ levels after a Neoproterozoic glaciation. Nature 453, 504-506.

BIGELEISEN, J., MAYER, M.G. (1947) Calculation of equilibrium constants for isotopic exchange reac tions. Journal of Chemical Physics 15, 261-267.

BLum, J.D SHERMAN, L S, JOHNSON, M.W. (2014) Mercury isotopes in Earth and Environmental Sciences. In: Jeanloz, R. (Ed.) Annual Review of Earth and Planetary Sciences. Vol 42. Annual Reviews, Palo Alto, 249-269.

CAO, X.B., LIU, Y. (2011) Equilibrium mass-dependent fractionation relationships for triple oxygen isotopes. Geochimica et Cosmochimica Acta 75, 7435-7445.

Clayton, R.N., Grossman, L., MAYEDA, T.K. (1973) Component of primitive nuclear composition in carbonaceous meteorites. Science 182, 485-488.

DeINES, P. (2003) A note on intra-elemental isotope effects and the interpretation of non-massdependent isotope variations. Chemical Geology 199, 179-182.

Eiler, J., Cartigny, P., Hofmann, A.E., Piasecki, A. (2013) Non-canonical mass laws in equilib rium isotopic fractionations: Evidence from the vapor pressure isotope effect of SF6. Geochimica et Cosmochimica Acta 107, 205-219.

FARQUHAR, J., BAO, H.M., THIEMENS, M. (2000) Atmospheric influence of Earth's earliest sulfur cycle. Science 289, 756-758.

Kaiser, J., Rockmann, T., BRenNinkmeijeR, C.A.M. (2004) Contribution of mass-dependen fractionation to the oxygen isotope anomaly of atmospheric nitrous oxide. Journal of Geophysical Research-Atmospheres 109, 11.

КотAKA, M., Окамото, M., Bigeleisen, J. (1992) Anomalous mass effects in isotopic exchange equilibria. Journal of the American Chemical Society 114, 6436-6445.

LI, S.N., LeVIN, N.E., CHESSON, L.A. (2015) Continental scale variation in O-17-excess of meteoric waters in the United States. Geochimica et Cosmochimica Acta 164, 110-126.

MATsuhisa, Y., GolDSMith, J.R., CLAYTON, R.N. (1978) Mechanisms of hydrothermal crystallization of quartz at $250^{\circ} \mathrm{C}$ and $15 \mathrm{kbar}$. Geochimica et Cosmochimica Acta $42,173-180$.

OtAKe, T., LASAGa, A.C., Онмото, H. (2008) Ab initio calculations for equilibrium fractionations in multiple sulfur isotope systems. Chemical Geology 249, 357-376

Sharp, Z.D., Gibbons, J.A., Maltsev, O., Atudorei, V., Pack, A., Sengupta, S., Shock, E.L. KNAUTH, L.P. (2016) A calibration of the triple oxygen isotope fractionation in the Sio2- $\mathrm{H} 2 \mathrm{O}$ system and applications to natural samples. Geochimica et Cosmochimica Acta 186, 105-119.

SKARON, S., WOLFSBERG, M. (1980) Anomalies in the fractionation by chemical-equilibrium of O-18O-16 relative to O-17-O-16. Journal of Chemical Physics 72, 6810-6811.

Swain, C.G., Stivers, E.C., ReuwER, J.F., SCHAAD, L.J. (1958) Use of hydrogen isotope effects to identify the attacking nucleophile in the enolization of ketones catalyzed by acetic acid. Journal of the American Chemical Society 80, 5885-5893.

UREY, H.C. (1947) The thermodynamic properties of isotopic substances. Journal of the Chemical Society, 562-581.

WOLFSBERG, M. (1972) Theoretical evaluation of experimentally observed isotope-effects. Accounts of Chemical Research 5, 225. 
Young, E.D., GaLy, A., NAgahara, H. (2002) Kinetic and equilibrium mass-dependent isotope fractionation laws in nature and their geochemical and cosmochemical significance. Geochimica et Cosmochimica Acta 66, 1095-1104.

Young, E.D., Kohl, I.E., Warren, P.H., Rubie, D.C., Jacobson, S.A., Morbidelli, A. (2016)

Oxygen isotopic evidence for vigorous mixing during the moon-forming giant impact. Science

351, 493-496. 